\title{
Out-of-Plane Scattering in Photonic Crystal Slabs
}

\author{
Wim Bogaerts, Student Member, IEEE, Peter Bienstman, Student Member, IEEE, Dirk Taillaert, Student Member, IEEE, \\ Roel Baets, Senior Member, IEEE, and Daniel De Zutter, Senior Member, IEEE
}

\begin{abstract}
Photonic crystal slabs combine a slab waveguide with an in-plane photonic crystal. Light is then confined in-plane by the photonic crystal and out-of-plane by the slab waveguide. The etched structures will cause light to scatter out of the waveguide plane. We studied the out-of-plane scattering losses using a two-dimensional approximation of this three-dimensional structure, with etched slots instead of holes. Our simulation techniques include mode expansion with perfectly matched layer absorbing boundary conditions. We show that the losses increase with higher index contrast, but that with very high-index contrasts light can be coupled into lossless Bloch modes.
\end{abstract}

Index Terms-Integrated optics, optical materials, optical planar waveguides, optical propagation in nonhomogeneous media, optical scattering.

\section{INTRODUCTION}

$\mathbf{O}$ NE of the most promising applications of photonic crystals today is the use of two-dimensional (2-D) crystal structures (e.g., air holes in a large index material) in combination with a layered waveguide to confine light in the third dimension [1], [2]. Light with wavelengths within the bandgap of the photonic crystal could be confined in a channel waveguide in the lattice (e.g., a missing row) or other types of defects [3], or light with wavelengths outside the bandgap could propagate as a Bloch mode of the periodic structure [4], [5]. In either case the light will feel the effect of the etched air holes, where there is no refractive index contrast in the vertical direction. These air holes can be a cause of radiation leakage from the slab waveguide's core to the cladding due to scattering of light. Good understanding of these losses could allow us to treat 2-D photonic crystal slabs as a 2-D problem, with out-of-plane scattering losses modeled as complex materials. This could reduce the requirements for 2-D photonic crystal slab modeling [6].

To keep the out-of-plane scattering low, two seemingly contradictory approaches have been suggested. The first approach aims for a conventional heterostructure, like the common GaAs- $\mathrm{Al}_{x} \mathrm{Ga}_{1-x} \mathrm{As}$ material system, with modest-to-low refractive index contrast between layers. Bénisty et al. suggest that out-of-plane scattering losses in these structures increase

Manuscript received January 3, 2001; revised February 21, 2001. This work was supported in part by the European Union in the context of the IST project PICCO and in the context of the Belgian DWTC project IUAP IV-13. The work of W. Bogaerts was supported in part by the Flemish Institute for the industrial advancement of Scientific and Technological Research (IWT) under a specialization grant. The work of P. Bienstman was supported in part by the Flemish National Fund for Scientific Research (FWO-Vlaanderen) under a doctoral fellowship.

The authors are with the Department of Information Technology (INTEC), Ghent University-IMEC, 9000 Gent, Belgium (e-mail: Wim.Bogaerts@intec.rug.ac.be).

Publisher Item Identifier S 1041-1135(01)04545-1.

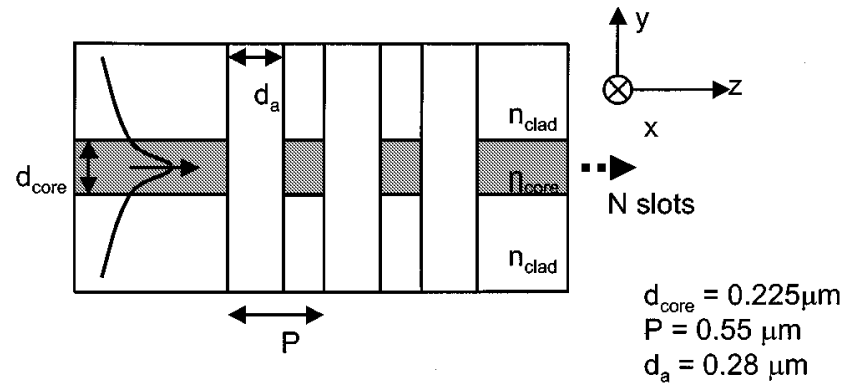

Fig. 1. Geometry of the simulated structure.

with the square of $\Delta \varepsilon$, where $\Delta \varepsilon=n_{\text {core }}^{2}-n_{\text {clad }}^{2}$, with $n_{\text {core }}$ and $n_{\text {clad }}$ the refractive index of the slab waveguide's core and cladding, respectively [1], [6]. This requires infinitely deep holes, or at least holes deep enough for the waveguide mode not to feel the bottom. Here, the best way is to keep the refractive index contrast as low as possible.

A different approach is to go for very high-index contrasts in the slab waveguide, up to semiconductor suspended in air. A more practical material could be silicon on insulator (SOI) with an index contrast of about 3.5-1.45. With these high contrasts, one can create periodic structures with Bloch modes well below the light line. For these modes, this means that no coupling to the radiation continuum is possible [4], [5]. However, they are lossless only in a perfect periodic lattice, whereas practical photonic crystal applications are finite and/or have defects where out-of-plane scattering can occur.

A 2-D approximation of the full three-dimensional (3-D) structure was studied. Our simulation structure consists of a three-layer slab waveguide with air slots etched through the structure, as illustrated in Fig. 1. We use this structure as an approximation of a channel waveguide in a 2-D periodic photonic crystal slab. This has the limitation that light cannot "flow around" the air holes as it would in the channel waveguide, and that therefore losses will be higher.

We will show here that by increasing the refractive index contrast between core and cladding of the waveguide structure, we will indeed observe an increase of scattering losses as predicted [1], [6], but that losses drop severely once a lossless Bloch mode is excited.

The remainder of this letter is structured as follows. In Section II, we briefly discuss the simulation tools we used for our calculations. Section III gives a detailed description of the simulated structure. Section IV describes the losses of a single air slot, which can be regarded as an incoherent source of out-ofplane scattering. In Section V, the results for multiple air slots are presented and we show how the coherent effects can reduce the out-of-plane scattering for high-index contrasts. 


\section{Simulation Techniques}

To calculate the characteristics of the optical structures under consideration, we used our simulation tool CAMFR [7], a vectorial eigenmode expansion tool with perfectly matched layer (PML) boundary conditions. The structure is divided into sections with a constant refractive index profile along the propagation axis, where the field is expanded into the eigenmodes of that section. The continuum of radiation modes is approximated by placing the structure between two perfectly conducting metal walls coated with PML to eliminate the parasitic reflections. At the interfaces between different waveguide sections, modematching is used to decompose the field into the eigenmodes of the new section, resulting in a scattering matrix for the entire structure. With this technique, we can calculate reflection and transmission of finite structures but also band structures for infinite periodic structures with the use of Bloch boundary conditions in the propagation direction. FDTD was used as a verification tool.

\section{Simulated Structure}

In our layer structure, we tried to approach semiconductor heterostructures, using a three-layer slab waveguide with a core thickness of $0.225 \mu \mathrm{m}$ and a refractive index $n_{\text {core }}$ of 3.5. The slab waveguide is a single mode at a wavelength of $1.55 \mu \mathrm{m}$ for all values of $n_{\text {clad }}$. We then vary the waveguide cladding index $n_{\text {clad }}$ to study its behavior for different values of $\Delta \varepsilon$. Some of these correspond to real layer structures. A maximum $\Delta \varepsilon$ of 11.25 represents a silicon membrane in air. A silicon core in an oxide cladding has a $\Delta \varepsilon$ of 10 , while a typical $\mathrm{GaAs}-\mathrm{Al}_{x} \mathrm{Ga}_{1-x} \mathrm{As}$ heterostructure has a $\Delta \varepsilon$ of about 2 .

\section{LOSSES IN A Single AIR SLOT}

The scattering of a single air slot can be treated as the incoherent limit where each hole radiates independently, thereby accumulating scattering losses in each hole or slot. This model was proposed by Bénisty et al. [1], treating holes as independently radiating dipoles.

Rigorous simulations of the losses of a single air slot confirm that out-of-plane scattering increases with higher refractive index contrast. For very low-index contrast the losses increase with the square of $\Delta \varepsilon$, as predicted by Bénisty et al. [6]. For higher contrasts, this relationship breaks down and the losses level out. When one is working with low-to-medium index contrast, one should keep the index contrast as low as possible, for a small increase in the cladding refractive index can yield a considerable drop in losses. This is not so for higher contrasts where a change in $\Delta \varepsilon$ has little effect on the out-of-plane scattering losses [8].

\section{LOSSES IN MUltiPle AIR SLOTS}

As photonic crystals are periodic structures, treating each air hole independently seems overly simplistic. It is shown that a 2-D periodic structure can support lossless Bloch modes, where the scattering of the holes interferes destructively [4].

We studied a periodic structure as in Fig. 1, with a period $P$ of $0.55 \mu \mathrm{m}$ and an air slot width $d_{\mathrm{a}}$ of $0.28 \mu \mathrm{m}$. We chose these (a) $\Delta \varepsilon=2.00$

$\mathrm{n}_{\text {clad }}=3.2$

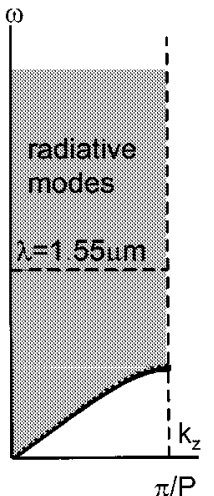

(c) $\Delta \varepsilon=10.92$

$\mathrm{n}_{\text {clad }}=1.15$

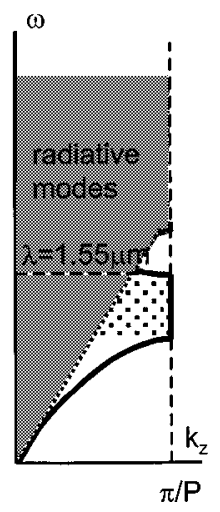

(b) $\Delta \varepsilon=10.00$

$\mathrm{n}_{\text {clad }}=1.5$

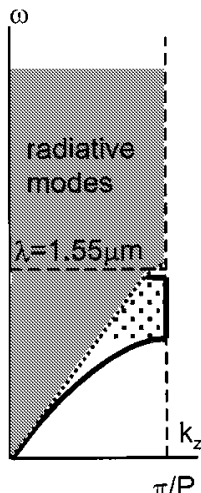

(d) $\Delta \varepsilon=11.25$

$\mathrm{n}_{\text {clad }}=1.0$

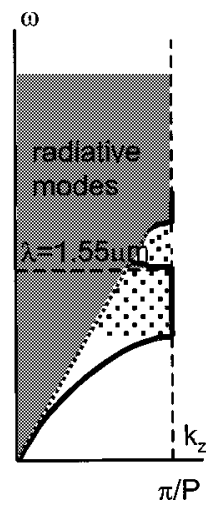

Fig. 2. Band diagrams of the structure in Fig. 1 for different values of $\Delta \varepsilon$.

parameters such that for a wavelength $\lambda$ of $1.55 \mu \mathrm{m}$ the periodic structure goes through four distinct regimes just by changing the refractive index contrast between core and cladding. Of course, a transition between these four regimes can also be brought about by changing other structure parameters. Fig. 2 shows the band structure of the infinite 2-D structure for these four regimes at different values of $\Delta \varepsilon$. The shaded region bounded by the light line indicates the continuum of the radiation modes. In Fig. 2(a) $\left(\Delta \varepsilon=2.0\right.$, typical for a $\mathrm{GaAs}-\mathrm{Al}_{x} \mathrm{Ga}_{1-x} \mathrm{As}$ heterostructure) we are obviously working outside of a bandgap and above the light line; therefore, we expect out-of-plane scattering losses to increase with increasing refractive index contrast, since the light can easily couple to the radiative continuum. These losses are plotted in Fig. 3 as a function $\Delta \varepsilon$. Because when working outside of the bandgap, the characteristics of a finite periodic structure are a nonmonotonous function of the number of periods, we took the average of the losses of the periodic structures with $30-50$ periods to eliminate these fluctuations.

For an index contrast of $\Delta \varepsilon=10.00$ [Fig. 2(b)] the infinite periodic structure has a region below the light line for high values of $k_{z}$. However, there is no guided mode present.

For a value of $\Delta \varepsilon=10.92$ [Fig. 3(c)] the band diagram shows a lattice mode below the light line at the operating wavelength. For the infinite periodic structure, this mode would have no radiation losses. This can be considered true for the bulk of a finite periodic structure. We see in Fig. 3 that losses drop sharply in 


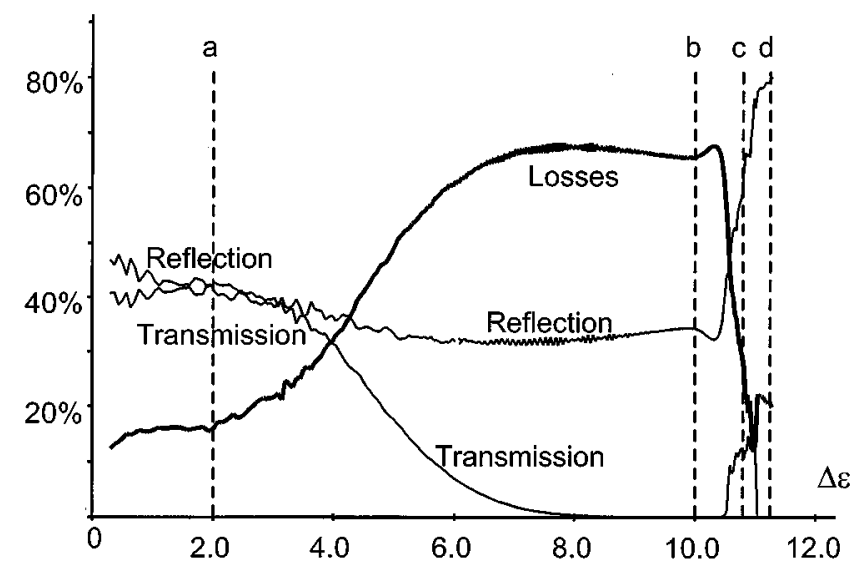

Fig. 3. Reflection, transmission and out-of-plane scattering loss for the structure of Fig. 1 as a function of refractive index contrast $\Delta \varepsilon$. Values are averages over a structure with $30-50$ periods to eliminate oscillations related to the finite number of periods.

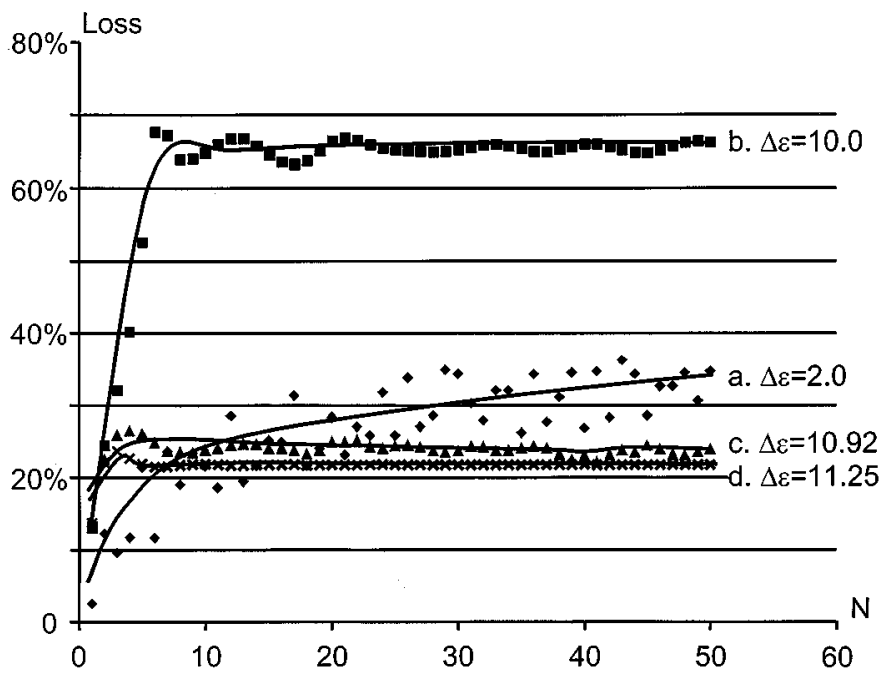

Fig. 4. Out-of-plane scattering losses as a function of number of periods $N$ for the four cases in Fig. 2.

this region, with a strong increase in transmission. Most of the losses occur at "defects"-in this case, the interfaces with the slab waveguide.

For even higher refractive indexes [Fig. 2(d)], we observe a photonic bandgap at $1.55 \mu \mathrm{m}$. Most of the incident light is therefore reflected in the first few periods. As a result, losses are also quite low in this regime and can again be credited to the boundary of the periodic structure.

The losses of these four structures as a function of the number of periods $N$ are plotted in Fig. 4, together with a broad moving average. As expected, for low-index contrast $(\Delta \varepsilon=2.0)$ we find low losses per slot, but the total loss increases as more slots are added. For a higher index contrast of $\Delta \varepsilon=10.00$ losses per slot are much higher, and we need only a few to lose all the light (the remaining light is reflected). For a guided Bloch mode, the only losses are "defect" losses at the lattice boundaries, so the loss is invariant with the number of periods. In the photonic bandgap most incident light is reflected within the first few periods, so losses remain constant as we add more periods.

\section{CONCLUSION}

Out-of-plane scattering limits the usefulness of 2-D photonic crystal slabs. It is therefore important to choose a good layer structure for keeping these losses low. We studied the out-ofplane scattering losses of a one-dimensional periodic structure with air slots. Four different regimes are observed for such a structure. For low refractive index contrasts, losses increase with increasing refractive index contrasts. For high contrasts, losses are very high unless one can excite a Bloch mode below the light line [5]. In a photonic bandgap, losses are low because light does not penetrate the crystal. Of course, by altering the photonic crystal's band structure using parameters other than $\Delta \varepsilon$, one can excite a guided Bloch mode for lower refractive index contrasts.

Summarized, two regimes are favorable to reduce undesired out-of-plane scattering, depending on the application. In a compact structure with many close-packed defects (cavities, bends, etc.) a low refractive index contrast is preferable, as in this regime defects scatter only a modest amount of light, as does each crystal period. For large structures with little or no defects, a lossless Bloch mode, only possible with high refractive index contrasts, is the obvious option.

In this letter, we presented infinitely deep slots. In real-life structures, the slot or hole depth is limited by etching capabilities. In the low-contrast regime, one will therefore have to balance between keeping a low-index contrast (with a broad waveguide mode) and the depth of the air slots to avoid additional scattering at the bottom of the air slots. In the high-index contrast regime, one can target a regime where the losses in the bulk of the periodic structure can theoretically be reduced to zero. However, applications using these structures will always require defects that will introduce losses in the otherwise lossless structure.

\section{REFERENCES}

[1] H. Bénisty, C. Weisbuch, D. Labilloy, M. Rattier, C. J. M. Smith, T. F. Krauss, R. M. De La Rue, R. Houdre, U. Oesterle, C. Jouanin, and D. Cassagne, "Optical and confinement properties of two-dimensional photonic crystals," J. Lightwave. Technol., vol. 17, p. 2063, Nov. 1999.

[2] T. F. Krauss, R. M. DeLaRue, and S. Brand, "Two-dimensional photonic-bandgap structures operating at near infrared wavelengths," $\mathrm{Na}$ ture, vol. 383, pp. 699-702, 1999.

[3] T. Baba, N. Fukaya, and J. Yonekura, "Observation of light propagation in photonic crystal optical waveguides with bends," Electron. Lett., vol. 35, pp. 654-656, Apr. 1999.

[4] S. G. Johnson, S. Fan, P. R. Villeneuve, J. D. Joannopoulos, and L. A. Kolodziejski, "Guided modes in photonic crystal slabs," Phys. Rev. B, vol. 60, pp. 5751-5758, Aug. 1999.

[5] E. Chow, S. Y. Lin, S. G. Johnson, P. R. Villeneuve, J. D. Joannopoulos, J. R. Wendt, G. A. Vawter, W. Zubrzycki, H. Hou, and A. Alleman, "Three-dimensional control of light in a two-dimensional photonic crystal slab," Nature, vol. 407, pp. 983-986, Oct. 2000.

[6] H. Bénisty, D. Labilloy, C. Weisbuch, C. J. M. Smith, T. F. Krauss, D. Cassagne, A. Béraud, and C. Jouanin, "Radiation losses of waveguidebased two-dimensional photonic crystals: Positive role of the substrate," Appl. Phys. Lett., vol. 76, pp. 532-534, Jan. 2000.

[7] P. Bienstman and R. Baets, "Optical modeling of photonic crystals and VCSEL's using eigenmode expansion and perfectly matched layers," Opt. Quantum Electron., to be published.

[8] W. Bogaerts, P. Bienstman, D. Taillaert, R. Baets, and D. De Zutter, "Out-of-plane Scattering in 1D Photonic Crystal Slabs," Opt. Quantum Electron., to be published. 